\title{
Voronoi-Based ISD and Site Density Characteristics for Mobile Networks
}

\author{
Anders Landström and Håkan Jonsson \\ Department of Computer Science, \\ Electrical and Space Engineering \\ Luleå University of Technology \\ Luleå, Sweden \\ \{anders.p.landstrom, hakan.jonsson\}@1tu.se
}

\author{
Arne Simonsson \\ Wireless Access Networks \\ Ericsson Research \\ Luleå, Sweden \\ arne.simonsson@ericsson.com
}

\begin{abstract}
Inter-Site Distance (ISD) is a common measure for characterizing the site density in a mobile network. However, obtaining a good estimation of the ISD for a real world network is not trivial since the physical layout is usually quite more complex than a perfect theoretical hexagonal grid, due to a number of unavoidable factors such as site availability and traffic density.

Voronoi diagrams have been suggested for approximating cells from network layouts, providing a method for partitioning the covered area into cells defined by the proximity to the given set of sites. This yields a framework for site coverage approximation based on the actual site distribution, rather than anderlying theoretical model.

We present a novel measure, based on Voronoi diagrams, for characterizing the site density of a cellular network and provide a comparison to the more traditional ISD measure. This measure improves capacity assessments and modeling of real networks.

Index Terms-Voronoi diagram, ISD, site density, wireless networks.
\end{abstract}

\section{INTRODUCTION}

The spatial structure of a cellular network is often described and modeled by a hexagonal tessellation where each hexagon corresponds to a cell in the network, i.e. the geographical area covered by one unique base station [1]. In reality however, the actual layout pattern of a network is irregular due to factors such as site availability, topography, and traffic density [2]. Statistical propagation distributions in real irregular cell plans can be well modeled in homogeneous hex simulation environments, with proper propagation variations such as shadow fading, indoor losses, and line-of-sight modeling. To achieve an accurate average path loss and degree of coverage problem, however, a good Inter-Site Distance (ISD) estimation for the network to model is of importance [3]. The same is true for capacity simulations, where the actual site density in the network to study is crucial since the number of sites defines the capacity per area unit $\left[\mathrm{Mbps} / \mathrm{km}^{2}\right]$. The ISD, or site-tosite distance, is well defined in a perfect hexagonal cell layout [4], but must be estimated for real irregular networks where no single unique site-to-site distance exists.

To overcome the inaccuracy of the classical hexagonal model when applied to real world cellular networks, Voronoi diagrams have been suggested for tessellation and cell system optimization [2], [5], [6]. In this work we present a novel measure for cellular network density, based on the Voronoi diagram of the base station layout. By considering the distribution of the site density, measured in sites per area unit, a good characterization of the network can be obtained, allowing more accurate capacity assessments. A hex-equivalent ISD can be calculated for simulation comparisons and evaluations of real networks, giving more realistic capacity results than with the traditional ISD measure. Simulated site displacements can be matched to the distribution of the obtained measure, or alternatively the network can be divided into more homogeneous sub-areas which can be evaluated separately.

In section II, we discuss ISD estimation based solely on distances between the sites. A framework for estimations of the ISD from Voronoi diagrams is then provided in section III, and simulation results are presented in section IV. Results for real world networks are presented in section $\mathrm{V}$, followed by the conclusion in section VI.

\section{INTER-Site Distance}

Cell sizes in a hexagonal tessellation are often characterized by site-to-site distance, or Inter-Site Distance (ISD), which is the Euclidean distance between two adjacent sites in the perfectly symmetrical structure. Hence, ISD is a common measure of cellular network density. A real world network layout, however, is usually not perfectly hexagonal. Thus, the ISD of a real network needs to be measured in terms of the actual distances between the sites.

Consider a network containing $n$ sites. A simple estimation of the ISD can be obtained by letting the average distance of the $k \in \mathbb{N}(k<n)$ closest sites characterize a cell. For each site $m=1,2,3, \ldots, n$, let the sequence $\left\{d_{m, i}\right\}, i=$ $1,2,3, \ldots, n-1$, denote the sorted distances to the other sites, so that $d_{m, 1} \leq d_{m, 2} \leq d_{m, 3} \leq \ldots \leq d_{m, n-1}$. The per-cell $k$-neighbor ISD for the simulated network, $I S D_{m, k}$ is then defined by

$$
I S D_{m, k}=\frac{1}{k} \sum_{i=1}^{k} d_{m, i}
$$

The whole network can then be characterized by the average ISD for the contained cells. That is, the $k$-neighbor ISD for 


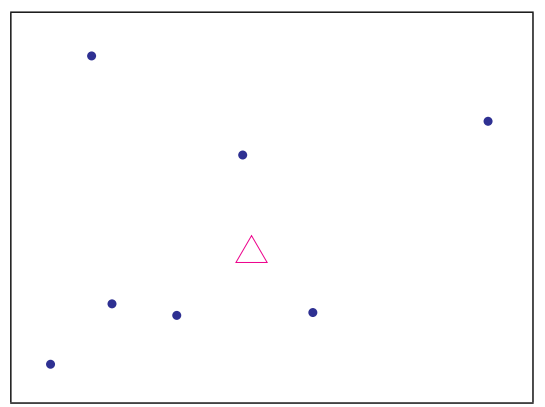

(a)

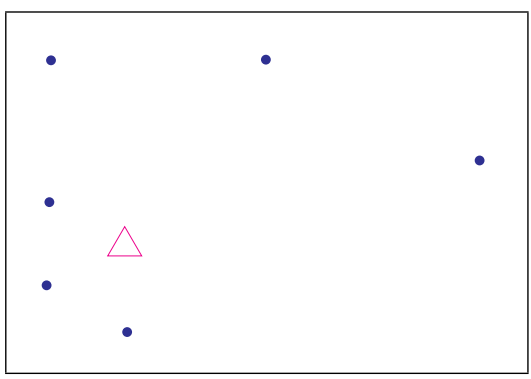

(b)

Fig. 1. Examples of $k>3$ being too high (a) and $k=3$ being too low (b) The triangle marks the site for which the ISD is being measured, while the dots mark other nearby sites.

the whole network, $I S D_{k}$, is given by

$$
I S D_{k}=\frac{1}{n} \sum_{m=1}^{n} I S D_{m, k} .
$$

Increasing $k$ takes distances to more of the surrounding sites into account, which may give a better characterization of the cell but risk including sites further away. Based on the assumption of an underlying hexagonal structure (where each cell has 6 neighbor cells), distorted due to practical deployment considerations and other environment-specific factors, it is reasonable to choose a $k$ such that $1 \leq k \leq 6$.

\section{Voronoi-Based Cell Density Characterics}

There may always be cases where $k>3$ yields an ISDmeasure influenced by non-neighboring sites - hence not well representing the actual network density if free space wave propagation is assumed. On the other hand; letting $k \leq 3$ for a network where distances between sites are more varying may result in an unreasonably low ISD measure. To see this, consider the examples in fig. 1 . In (a), $k=3$ would be more representative than $k=4$, and in (b), $k=3$ is too low to capture the surrounding network structure.

An alternative characterization of the network density can be extracted from the Voronoi diagram defined by the site positions. Let $\|x-y\|_{2}$ (the $L_{2}$-norm) denote the Euclidean distance between two points $x$ and $y$ in the plane and let $X=$ $\left\{x_{1}, x_{2}, x_{3}, \ldots, x_{n}\right\}$ denote a set of $n$ points. The Voronoi diagram is then defined as a subdivision of the plane into $n$ corresponding polygonal regions $P_{i}, i=1,2,3 \ldots, n$, such that for each point $y \in P_{i}$

$$
\left\|x_{i}-y\right\|_{2}<\left\|x_{j}-y\right\|_{2}, i, j=1,2,3 \ldots, n, j \neq i .
$$

This yields a plane tessellation based on connected convex polygons around the points in the defining point set $X$, where any point within a polygon $P_{i}$ is closer to the corresponding point $x_{i}$ than to any other points in $X$ [7]. Assuming that radio signal strength drops in relation to distance, we can let $X$ represent site locations and consider the Voronoi regions to be network cells [5].

\section{A. Voronoi-Based ISD}

Using a Voronoi tessellation constructed from site positions, we define a per-cell ISD by letting the average distance to the neighboring sites (which are well defined in the Voronoi diagram) represent the cell in question. That is, $k$ in (1) is allowed to vary from site to site so that it equals the number of neighboring cells in the Voronoi diagram, thereby allowing for a generalization of the fixed $k$-neighbor ISD measure under the assumption that the $k$ neighboring sites in the Voronoi diagram are the $k$ closest points. This strategy takes the surrounding network structure into account, without allowing distances to non-neighboring sites to effect the result. The average value $I S D_{V}$ of the per-cell Voronoi ISDs,

$$
I S D_{V}=\frac{1}{n} \sum_{m=1}^{n} I S D_{m, k(m)},
$$

can then be used to represent the whole network, allowing us to compare distances in the Voronoi diagram to ISD. Here $k(m)$ denotes that the number of neighboring sites considered is individual for each site $m$.

\section{B. Voronoi-Based Site Density}

From a Voronoi tessellation, it is possible to extract a network cell area for each site (assuming that signal strength drops in relation to distance to the site in question) [5]. This yields a measure which is related to the actual expected service area of the sites. By considering the areas of the cells (rather than just distances between neighboring sites) when characterizing the network a more complete description can be achieved, given that the Voronoi diagram is a reasonable estimation of the actual cell sizes. The area served by each cell is important for capacity evaluations, where traffic load is often defined as traffic density per area unit $\left[\mathrm{Mbps} / \mathrm{km}^{2}\right]$.

A per-cell area measure is retrieved from the Voronoi diagram by considering the area of the convex polygon originating from each site $m$, which can then be averaged for the studied part of a network. That is, the per-cell Voronoi cell area $V C A_{m}$ is given by

$$
V C A_{m}=\operatorname{Area}\left(P_{m}\right),
$$

where $P_{m}$ is the polygon in question, and the average Voronoi cell area $V C A$ for $n$ cells is given by

$$
V C A=\frac{1}{n} \sum_{m=1}^{n} V C A_{m} .
$$




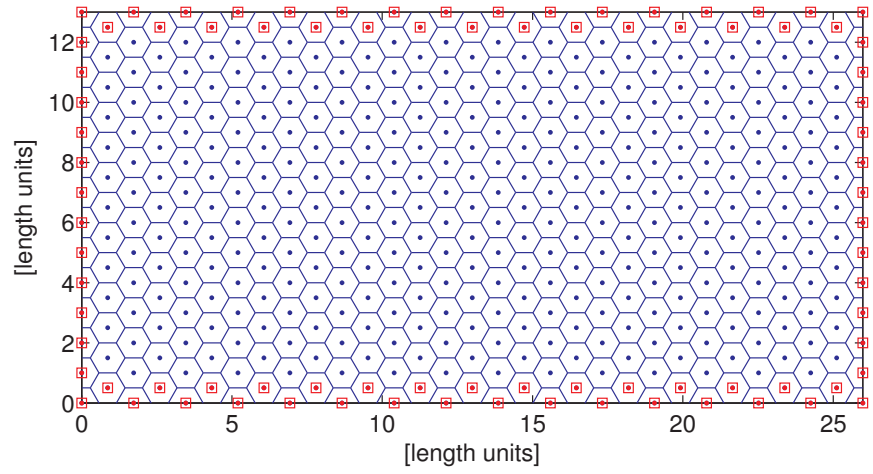

(a)

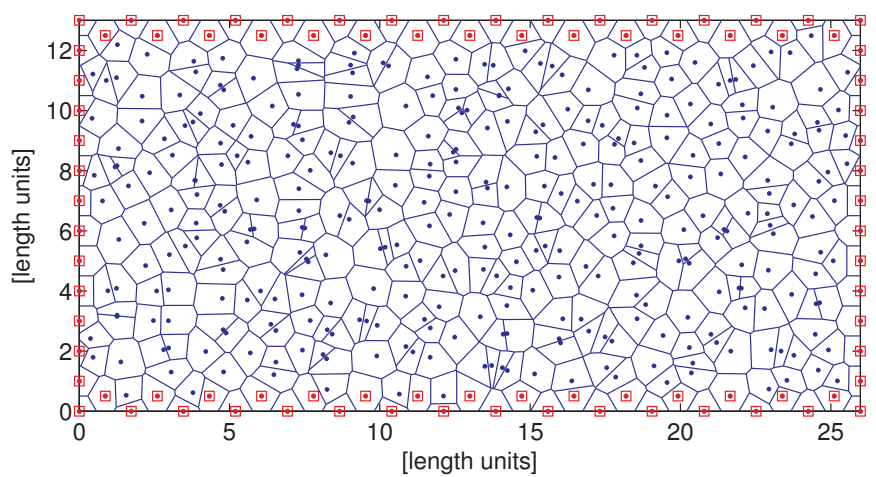

(b)

Fig. 2. Simulated network layouts: An original theoretical site layout with its hexagonal tessellation (a) and a simulated displacement of the sites with the corresponding Voronoi tessellation (b). Boundary sites are marked with squares.

The Site Density (SD) of the studied network can then be estimated, in sites per area unit, as

$$
S D=\frac{n}{\sum_{m=1}^{n} V C A_{m}}=\frac{1}{V C A}
$$

and a per-cell Site Density $S D_{m}$ for cell $m$ (corresponding to site $m$ ) can be defined by letting $n=1$, obtaining

$$
S D_{m}=\frac{1}{V C A_{m}} \text {. }
$$

For easier comparison towards the ISD measures, consider the area

$$
A_{\mathrm{hex}}=\frac{\sqrt{3}}{2} d^{2}
$$

of a hexagonal cell in a perfect grid with ISD $d$. Solving for $d$ while requiring $A_{\text {hex }}=V C A$, we obtain

$$
d=\sqrt{\frac{2}{\sqrt{3}} V C A} .
$$

We define $d$ in (10) to be the hex-equivalent ISD, which is the ISD required for an accurate area estimation in a simulated hexagonal grid. The hex-equivalent ISD can be used for comparing the SD measure to ISD.

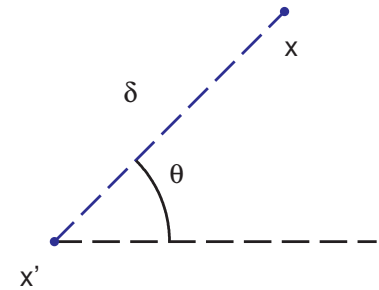

Fig. 3. Displacement example.

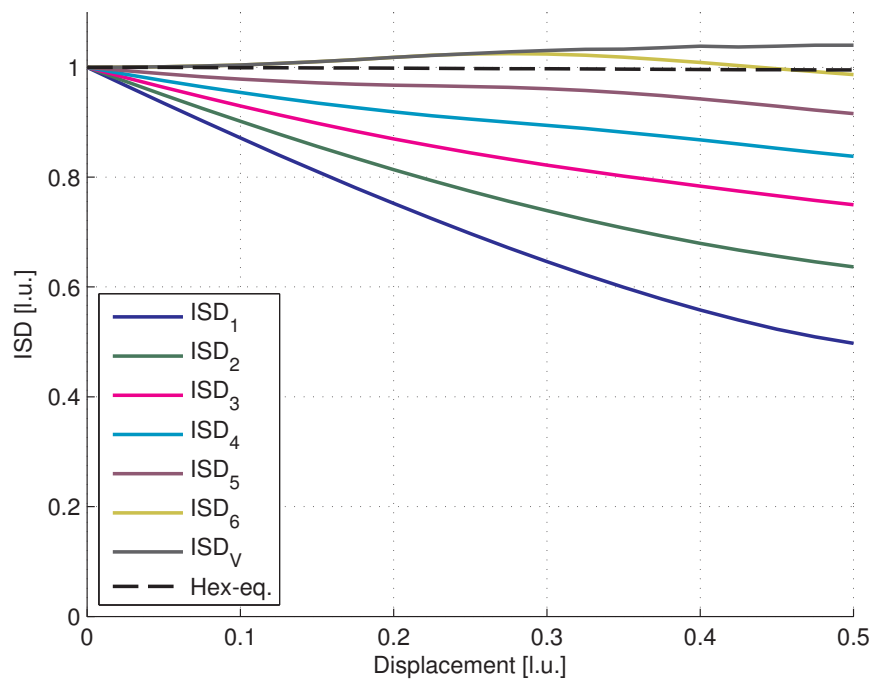

Fig. 4. Simulation Results

\section{Simulation}

The performance of the network density measures discussed in sections II and III can be evaluated and compared by applying them to a gradually deformed network layout. The original simulated cell layout is perfectly distributed with equal distance (= 1 length unit) between all neighboring sites, resulting in a hexagonal cell structure as presented in fig. 2a. Sites are divided into the two categories boundary sites and inner sites, the difference being that cells of boundary sites are touching the outer region border while cells of inner sites are not. We let $n$ in (2), (4), (6), and (7) denote the inner sites. Hence, ISD measures of boundary sites are not considered in the simulation results; these sites merely serves as an outer barrier restricting the Voronoi regions of the inner sites.

The layout is gradually deformed by introducing an increasing displacement $\delta$ for each original inner site location $x^{\prime}$ in a random direction $\theta$ (individual for each site), resulting in a new site location $x$ as shown in fig. 3 .

The ISD was estimated for the deformed networks, using the presented methods. The results, presented in fig. 4, clearly shows that a fixed $k$ will indeed result in a lower ISD measure than the generalization provided by the Voronoi ISD, where $k$ is allowed to vary between cells. Looking strictly at the $k$ closest neighbors, without respect to the actual surrounding network layout, will introduce a bias towards 


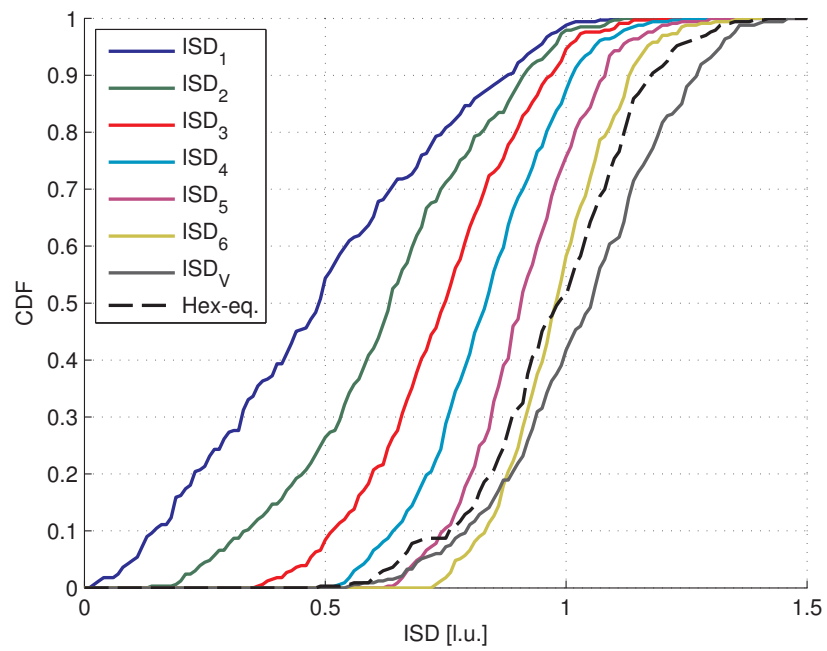

Fig. 5. CDFs for the different ISD estimations for displacement 0.5.

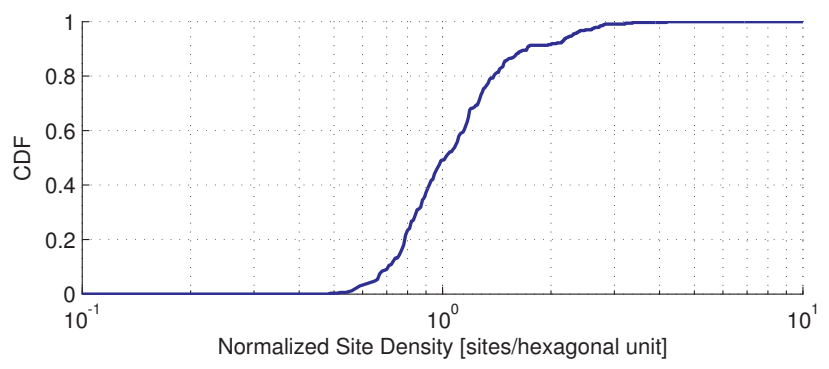

Fig. 6. $\mathrm{CDF}$ for Voronoi area distribution of the layout shown in fig. $2 \mathrm{~b}$.

low ISD measures - in particular for low values of $k$. Apart from the problems on a single cell basis earlier discussed, this will result in a systematic underestimation of the distances to neighboring sites, as compared to the Voronoi results which rely on the exact number of neighbor sites defined by the proximity measure given by (3).

As could be expected, the 6-neighbor ISD and the Voronoi ISD are identical until the displacement grows large enough to cause cells to switch neighbors. Cells that end up with more than six neighbors explain most of the difference between the the two curves for larger displacements, which further suggests that a fixed- $k$ ISD cannot capture these local characteristic properties.

Neglecting the minor changes of the total cell area of inner sites near the boundary sites, any ISD estimation intended for area calculations in homogeneous hex simulations should remain constant despite the random displacements since neither the number of sites in the region nor the total area of the region changes. In fact, any error in the ISD will be enhanced quadratically in (9) if the ISD is converted into an area. For instance, the 3-neighbor ISD would give more than $40 \%$ decrease in average area for a displacement of 0.5 , even though the ratio of number of sites over the total area is clearly the same as before the displacement. It is clear from fig. 4 that the only estimated ISD which would not lead to an erronous

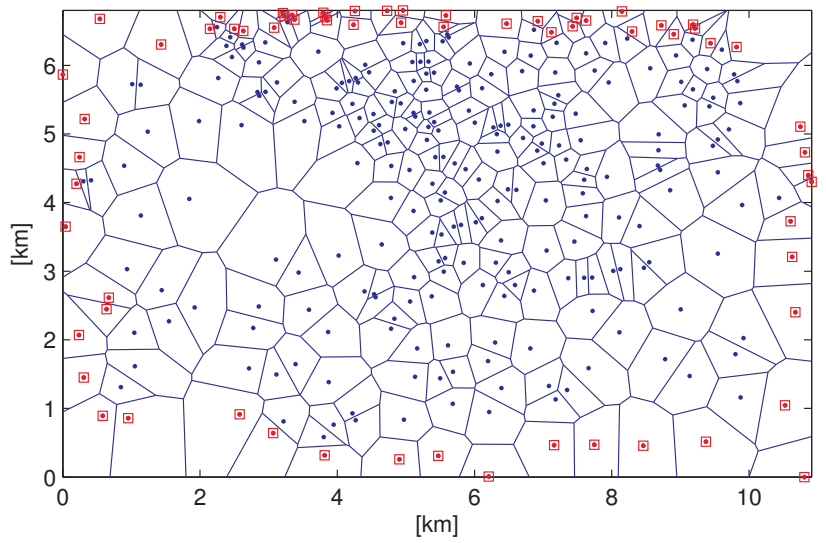

(a)

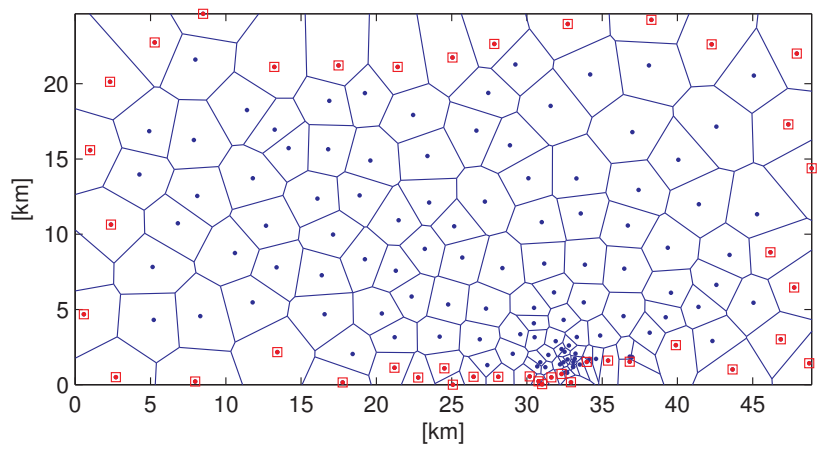

(b)

Fig. 7. Network layout examples A (a) and B (b). Boundary sites are marked with squares.

area calculation is the hex-equalized. Fig. 5 shows the case with displacement 0.5 in more detail, providing cumulative distribution functions (CDFs) for the presented ISD estimators. Here as well, it is clear that a fixed- $k$ ISD with a low $k$ has a bias towards low ISD estimations.

Once the Voronoi area approach has been validated towards the ISD measures, we can study cell sizes in the network directly by considering the distribution of the Site Density obtained from the Voronoi cell area by (8). An example is provided in fig. 6 , where SD has been normalized to sites per hexagonal unit, which is defined as the area of a hexagon in the original layout (with displacement 0 ).

\section{NETWORK CHARACTERIZATION}

In this section we present results from network characterizations by the proposed Site Density measure. Fig. 7 shows the layouts for two real world networks with different cell size distributions, and their average values for the different density measures are presented in Table I together with results for three other real world network layouts C, D and E. The more detailed Site Density distributions are presented in fig. 8. Network A (fig. 7a) consists of cells in a range of smaller sizes, which is clearly visible in the corresponding CDF. Network B (fig. 7b), on the other hand, is more dominated by larger cells but also has regions covered by smaller cells. Networks C, D, 
TABLE I

ESTIMATED MEASURES FOR REAL NETWORKS

\begin{tabular}{l|ccccc} 
Measure & A & B & C & D & E \\
\hline$I S D_{1}[\mathrm{~km}]$ & 0.25 & 1.91 & 0.73 & 0.14 & 5.17 \\
$I S D_{2}[\mathrm{~km}]$ & 0.30 & 2.07 & 0.82 & 0.17 & 6.03 \\
$I S D_{3}[\mathrm{~km}]$ & 0.34 & 2.25 & 0.90 & 0.20 & 6.74 \\
$I S D_{4}[\mathrm{~km}]$ & 0.38 & 2.41 & 0.98 & 0.22 & 7.37 \\
$I S D_{5}[\mathrm{~km}]$ & 0.42 & 2.58 & 1.06 & 0.24 & 7.91 \\
$I S D_{6}[\mathrm{~km}]$ & 0.45 & 2.73 & 1.13 & 0.26 & 8.42 \\
$I S D_{V}[\mathrm{~km}]$ & 0.49 & 2.74 & 1.16 & 0.29 & 8.90 \\
Hex-eq. $[\mathrm{km}]$ & 0.51 & 2.92 & 1.16 & 0.31 & 9.14 \\
\hline$V C A\left[\mathrm{~km}{ }^{2}\right]$ & 0.23 & 7.39 & 1.16 & 0.08 & 72.38 \\
$S D\left[\right.$ sites/km $\left.{ }^{2}\right]$ & 8.22 & 0.75 & 1.12 & 24.66 & 0.03
\end{tabular}

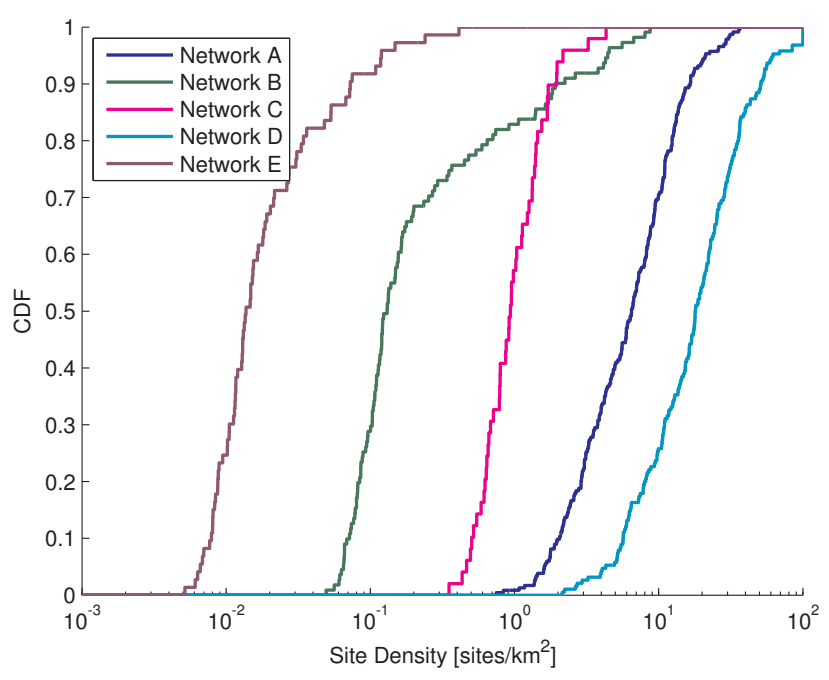

Fig. 8. Network layout example.

and $\mathrm{E}$ are examples of a medium, a high and a low density network, respectively.

The network characterization and suggested measures can be used in several different ways. The site density distribution indicates if a network can be statistically studied as a unit or if it should be divided. For example, networks A, C, and D are relatively uniform and may be studied using a regular hexgrid network with an ISD equal to the overall network hexequivalent ISD. However, the larger variances in site density for networks B and E indicate that these networks should be divided into more homogeneous sub-areas studied in separate hex-grid simulations. Otherwise important characteristics in different areas, such as the high capacity in the most dense parts, will be averaged out and hidden. Alternatively, displacements can be added to a hex-grid simulation environment, for example as in fig. 2, to create a site density distribution matching the studied network. Equation (6) gives a site density measure for each site, making it easy to identify sub-areas with different characteristics, and the average site density can be easily calculated for different sub-sets of sites. This can be considered more handy than drawing polygons for each alternative, calculating areas and counting sites.

\section{CONCLUSION}

Compared to an ISD calculated from a fixed number of neighbors, the distances and neighbor properties of the Voronoi diagram are more adaptive to the actual proximity relations between sites in a cellular network.

We have presented a Voronoi-based ISD measure, which generalizes ISD estimations based on a fixed set of neighbors, thereby providing a bridge between the two concepts. Voronoi diagrams can be used even better when considering cell areas however, and indirectly provides a Site Density measure. An accurate Site Density measure is important for network capacity assessments, and the corresponding hex-equalized ISD is useful for setting up a simulation environment giving accurate performance results. This measure does not cause errors in area estimations, and provides an even more complete description of a studied network.

\section{REFERENCES}

[1] T. Rappaport, Wireless Communications: Principles and Practice, 2nd ed. Upper Saddle River, NJ: Prentice Hall, 1996.

[2] A.-E. Baert and D. Seme, "Voronoi mobile cellular networks: topological properties," in 3rd International Symposium on Parallel and Distributed Computing (ISPDC), July 2004, pp. 29-35.

[3] A. Simonsson, M. Johansson, and M. Lundevall, "Antenna and propagation parameters modeling live networks," in 2011 IEEE Vehicular Technology Conference (VTC '11 Fall), Sept. 2011, pp. 1-5.

[4] 3GPP, TS 36.211, "Evolved universal terrestrial radio access (e-utra); further advancements for e-utra physical layer aspects (release 9)," 3GPP, Tech. Rep., 2010.

[5] M. Sengoku, H. Tamura, S. Shinoda, and T. Abe, "Graph \& network theory and cellular mobile communications," in 1993 IEEE International Symposium on Circuits and Systems (ISCAS '93), vol. 4, May 1993, pp. 2208-2211.

[6] F. Baccelli and S. Zuyev, "Stochastic geometry models of mobile communication networks," in Frontiers in Queueing. CRC Press, 1997, pp. 227-243.

[7] M. De Berg, O. Cheong, M. Van Kreveld, and M. Overmars, Computational geometry: algorithms and applications, 3rd ed. New York: Springer, 2008. 